\title{
The study on the application of virtual reality technology in the three-dimensional courseware
}

\author{
Xiaoqiang $\mathrm{Hu}^{1}$ Xinting $\mathrm{Wang}^{2}$ and Ling $\mathrm{He}^{3}$
}

\begin{abstract}
The virtual reality technology (referred to as VR) is the application of IT technology represents the last decade. It has powerful interactive features, give a visual representation of the three-dimensional information and content is widely used in the Products presentation, skill straining and other aspects. Construction teaching courseware based on virtual reality technology, using Virtools as a development tool and taking camera studying for example, to be further elaborated key technologies of three-dimensional modeling and dynamic interaction. It can greatly increase the learner's understanding of the content, and expand the effective supplement of current 3D courseware.
\end{abstract}

Keywords: Virtual Reality; Courseware; Virtools

\subsection{Introduction}

Virtual reality technology is based on computer technology, utilization and integrated 3D graphics technology, multimedia technology, simulation technology, sensor technology, display technology and other kind of high technology, using the computer and other equipments to generate a realistic three-dimensional visual, tactile, olfactory and other sensory experiences in the virtual world. [1] Then the people in the virtual world have a feeling of be personally on the scene. Virtual reality, network and multimedia technology are known as the three major technolo-

\footnotetext{
${ }^{1}$ Xiaoqiang $\mathrm{Hu}(\bowtie)$

Jiangxi Science \& Technology Normal University Nanchang, China

e-mail: chinavrmm@163.com

${ }^{2}$ Xinting Wang $(\bowtie)$

Jiangxi Science \& Technology Normal University Nanchang, China

e-mail:shuihuizj@163.com

${ }^{3}$ Ling He $(\bowtie)$

Jiangxi Science \& Technology Normal University Nanchang, China

e-mail: lynlynhe126@126.com
} 
gies of application prospect in 21 century. It has been widely used in military exercise, sports training, medical, commercial and other fields.

The rapid development of science and technology lead to major progress for our learning. The traditional blackboard gradually become a "whiteboard", simple courseware from plain text to multimedia forms, including text, sound, graphics, images, three-dimensional animation and so on. The teaching methods are continuously enriched. Human information perception about $80 \%$ is obtained through the eyes. Richard E.Mayer (1997) found that learners produce 50\% more creative solutions if they touch the combination of text, graphics, animation and other media presentation. New opportunities and challenges are developed for teaching methods because of the frontier virtual reality technology progress. Introduction virtual reality technology to the courseware, it provides learners a strong cognitive experience, which not only help to strengthen the abstract knowledge and enhance the ability to understand, but also rich expression of the media, and more interactive.

\subsection{The development tool-Virtools}

Virtools is a virtual reality engine, integrated 2D graphics image, 3D model, audio, video and other kinds of file format of interactive software, developed by France Dassault group's affiliated global interactive 3D development solutions company Virtools. It can be used for the virtual display, game development, construction navigation, and so on. Virtools itself has more than 500 Building Blocks (referred to as BB).Users create script on character or object with Building Blocks. The Building Blocks in a flowchart are determined in chronological order, using visual scripting language to create an interactive virtual world. The biggest characteristic is to meet the designers without program background and the senior program designers at the same time. Ordinary designers develop simple and efficient works through using Building Blocks; senior program designers can use the VSL (virtools scripting language) and SDK (software development kit) for further program design and product development, to create a variety of virtual reality world.

The works of Virtools can be outputted the format of web pages, and further edited, integrated with general web pages or flash. So that the audience can view interactive virtual reality world in the ordinary web page, allowing people interact with the works directly. Virtools has powerful Render Engine, supporting a variety of textures. It use more advanced picture rendering technology, making the virtual experiment system is more realistic in the situation. The strong visual impact and rich experimental situation, can greatly improve the students' learning initiative. 


\subsection{Research on 3D courseware based on Virtools}

There are some steps to make three-dimensional courseware by Virtools. Step 1: According to the practical needs to build three-dimensional models by using modeling software, which is the foundation of virtual and vivid, realistic, but also one of the key to attract learners. Step 2: In addition combine using the BB module, it is also possible write $\mathrm{BB}$ module by $\mathrm{C}++$, gradually creating a complete virtual experiment system. Step 3: It is necessary to correct and evaluate the system in order to identify errors and deficiencies in the program flow. This is the important part of the virtual experiment production process but also a guarantee of quality. Step 4: The works of Virtools is outputted the format of web pages, allowing the learners view interactive virtual reality world online or in the laboratory.

\subsubsection{Creating the models}

It is the first way to creating models before construction of virtual experiment. Virtools software itself does not have the function to create model, therefore, so the model was established by 3Ds Max. [2] The software ownership is big and the reference material is easy to obtain. But there need special software or plug-in to transformation the format. [3] On the one hand it is important to consider the reliability requirements, the more fine the more lifelike; on the other hand, the model is too large to interaction response slowly, affecting real time requirements. Altogether, simplify the model in order to ensure visual and interaction. Such as reduce the face of model, delete the history record after model creation, elect the textures' format correctly. As shown in figure 1.1

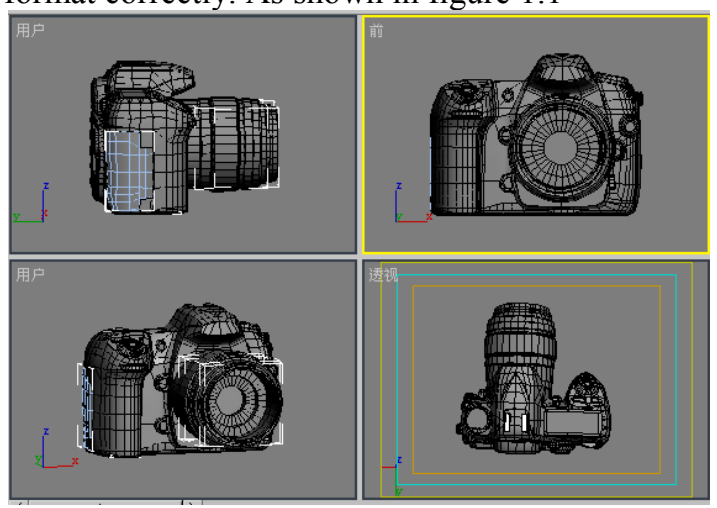

Fig. 1.1 The 3D model of camera 
After completing the model, rendering output the format of .nmo. The format's outputting need install the Max To Virtools plug-in. Then the model is imported into Virtools and designed interaction

\subsubsection{Realization the dynamic interaction}

Learning situation analysis is the key of the virtual experiment design. The designed experiments should be centers around the learner, which is a "learnercentered" teaching idea. The purpose is to understand the learner readiness and learning styles, so that teaching students in accordance with their aptitude.

\subsubsection{Camera setting}

Cameras like the eyes of the players. There are two ways to set cameras. [4] (1) The first person camera: from the perspective of the rover to get roaming experience in the virtual scene. As shown in figure 1.2. (2) The following camera: cameras follow the objects in the virtual scene or to achieve the function of the third perspective. In this paper, we select the following camera, so that learners can observe objects from any angles. As shown in figure 1.3.

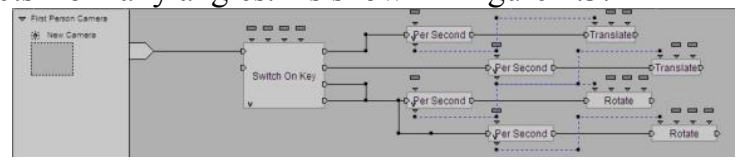

Fig. 1.2 The first person camera scripts

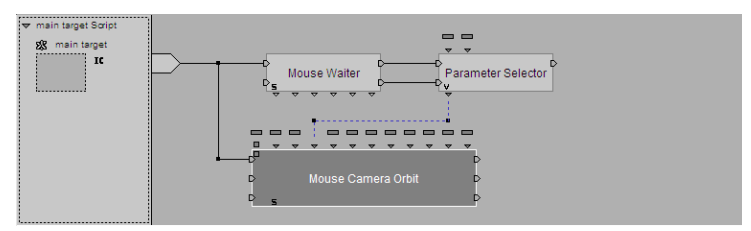

Fig. 1.3 The following camera scripts

\subsubsection{Scene manage}

The enrichment scene can improve visual effect and fully show the interaction. By setting the Push Button parameter, realize the jump page. [5]The script was shown in figure 1.4. When the scene is excessive, using scene management technology provided by Virtools, namely, the complicated procedures divided into 
a plurality of scenes to unified management. As result, one scene that can be in active state, which reducing the script engine burden and making the project run more smoothly
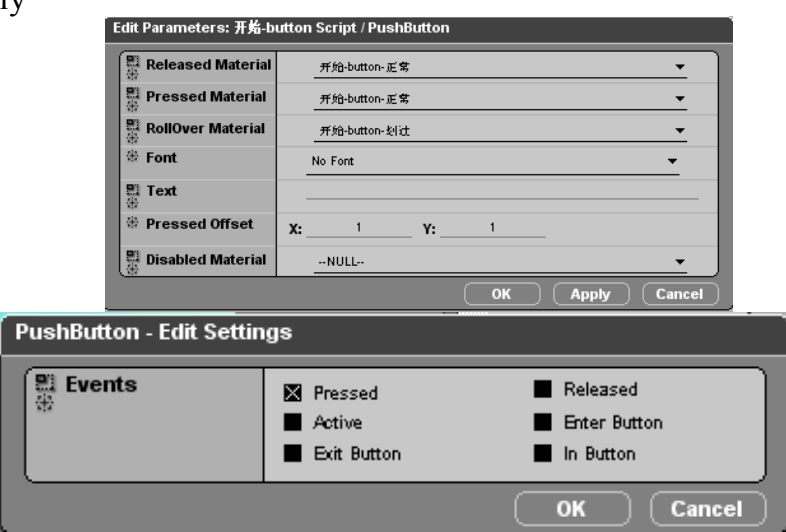

Fig. 1.4 The parameter setting of PushButton

\subsubsection{The interactions of three-dimensional objects -- select, move and ro-} tate

In order to get plenty experience, the system provides interactive experience with user and objects. For example, when the left mouse button to choose the USB cover of virtual camera model, the cover can rotate and open. This procedure can be generally divided into two steps: the not press state and press state. Using 2D Picking BB click on the camera parts, through the Send Message and Wait Message module to control the information sending and receiving.[6] As shown in figure 1.5.

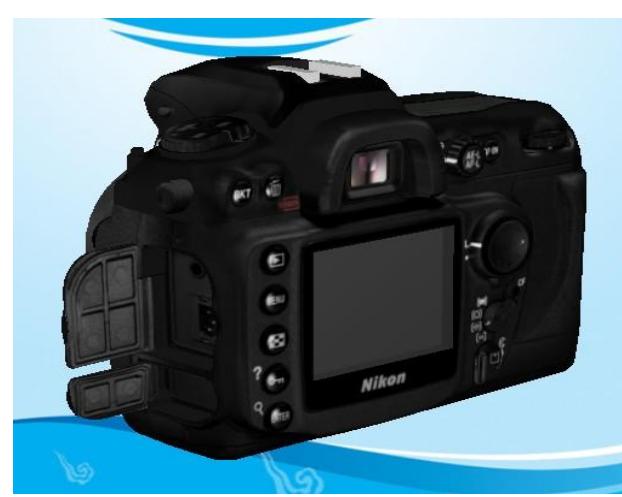

Fig. 1.5 The result of select, move and rotate 


\subsubsection{Video switching control}

This main function provides the camera history introduction. During playback process the learner can have the freedom control. Set video through Texture to play. The video load and control use Video Input Properties and Video Player. Mouse Waiter、2D Picking control the button response. Switch On Parameter control the video's play, pause and stop. The description was shown in Figure 1.6.

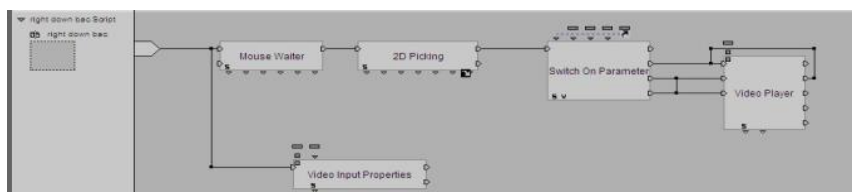

Fig. 1.6 Video switching control

\subsubsection{Camera function realization}

In the experiment of focal length adjustment, click different focal length button, the scene will switch to the current focal length scene automatically, achieving the result of the pictures. In order to save time and simulate real world, the background picture and the showing pictures were shot in the real scene. The focal lengths are $18 \mathrm{~mm}, 24 \mathrm{~mm}, 35 \mathrm{~mm}, 45 \mathrm{~mm}$ and $55 \mathrm{~mm}$, importing to Virtools at the same time. Adding the $\mathrm{BB}$ of mouse events and mapping texture to achieve the different effect with different focal length button switching. The same as aperture adjustment and shutter speed experiments. As shown in figure 1.7 and 1.8.

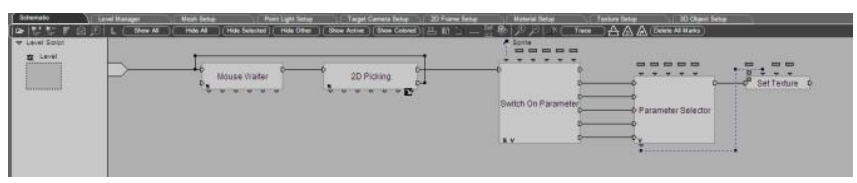

Fig. 1.7 Camera control scripts

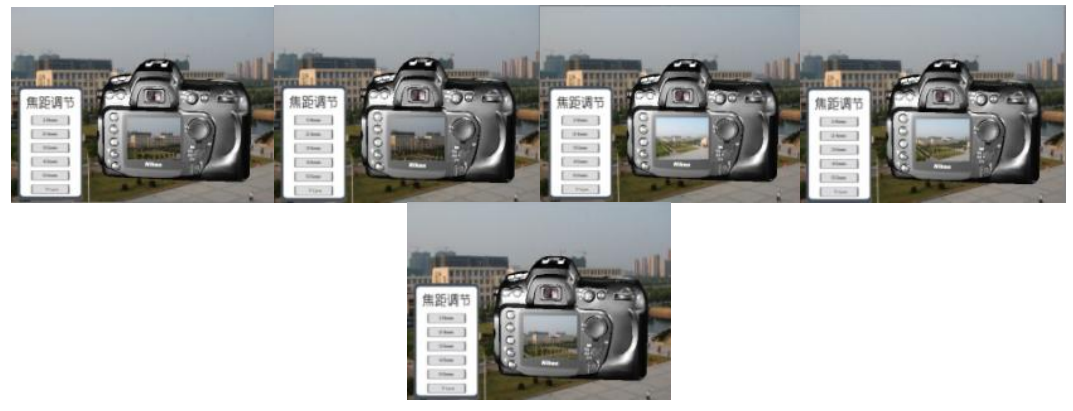

Fig. 1.8 Under the different focal length shooting effect 


\subsubsection{Collision detection}

The virtual characters or objects may knock against in the environment or objects of the environment. It may appear an unreasonable phenomenon of through objects. Then you need to add the BB to prevent the collision happen.There are three ways of Virtools collision detection. One setting collision is to add the Floor attribute or Fixed Obstacle attributes. The other one is the Group collision. Put obstacles into Group, such as walls, pillars, then add Prevent Collision and Object Slider BB to role. The Prevent Collision do not have sliding function, so it will appear angle jam problem in the process of collision, then we use Object Slider setting sliding. The last way is the Grid collision; mainly through the edit mesh cover the obstacles, using the Layer Slider BB to avoid the collision. Grid is hidden after the settings, but the collision detection function did not disappear. In actual operation, it often combining uses the first and second methods. The third methods, it must ensure the size of the grid setting properly, so that the obstacle region covered with the actual object size. If the coverage area is set too large or too small, it can not correctly detect collision.

\subsubsection{The font settings}

Virtools provides font system that the system font generates in 2D Frame to display, by using the Create System Font, Set Font Properties and 2D Text BB. In the Set Font Properties, it can set the character spacing, font scaling, inclination, font color, which can be modified according to personal preferences. The information goes into 2D Text BB with English. If you want to display Chinese by using the Text Display BB.

\subsubsection{Work publishing}

When all programs are completed, it can be published. Using HTML web publishing form, and generate.Vmo format file at the same time. Note that, the web page's file path and file name can not use Chinese, otherwise it will lead the file could not play normally.

\subsubsection{Conclusion}

With the continuous development of virtual technology and the application of stereo projection technology in the teaching field, Virtools can be applied in 
Vrpack to make the released virtual experimental teaching system works put on the screen in the method of stereographic projection. [7]Compared with the traditional teaching mode, it has the nature of image, intuition, and immersion and it can greatly improve the quality and efficiency of teaching. At the same time, the use of virtual reality technology combined with network, learners can through the network access to three-dimensional courseware. This way will make a qualitative leap in the design of courseware, that has an extensive space in long-distance education and it is a new direction in future learning

\subsection{References}

1. Hu Xiaoqiang, virtual reality technology foundation and application [M]. Beijing: Beijing University of Posts and Telecommunications Press(2009)

2. Fu Zhiyong, Gao Ming. Three Dimensional Game Designs by Virtools Dev 4.0 [M] Beijing: Tsinghua University Press (2008)

3. Liu Mingkun. 3D game designer's bible: Virtools' development tools article [M]. Sichuan: Sichuan Electronic Audio and Video Publishing House(2005)

4. Wang Liqun, Li Hongsong. Computer games plan and design: Virtools' concise course [M]. Shanghai: Fudan University Press (2008)

5. Liu Mingkun. 3D game designer's bible: Virtools' game developers actual combat [M]. Guangdong: Shantou University Press (2006)

6. Liu Mingkun. VT game design secret recipe [M] Beijing: China Youth Press, (2010)

7. Ruan Lina. Research and Design of Computer Hardware Virtual Experiment Based on Virtools for High School [D]. Sichuan Normal University (2012) 